\title{
Nucleation-Mediated Lateral Growth on Foreign Substrate
}

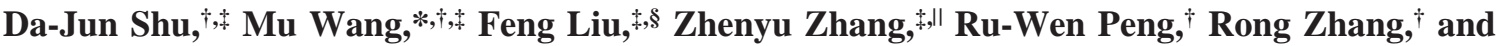 \\ Nai-Ben Ming ${ }^{\dagger}$ \\ National Laboratory of Solid State Microstructures and Department of Physics, Nanjing University, Nanjing \\ 210093, China, International Center for Quantum Structures, Chinese Academy of Sciences, Beijing 100080, \\ China, Department of Materials Science and Engineering, University of Utah, Salt Lake City, Utah 84112, and \\ Solid State Division, Oak Ridge National Laboratory, Oak Ridge, Tennessee 37831
}

Received: August 3, 2006; In Final Form: December 1, 2006

\begin{abstract}
A model is developed to deal with lateral growth of a crystalline layer on a foreign substrate, which is mediated by successive nucleation at the concave corner defined by the meeting of a crystal facet and the substrate. It is demonstrated that due to an imbalance of surface/interface tensions at the concave corner, once the embryo of a nucleus is formed, the crystallographic orientation of the nucleus is spontaneously twisted. By successive nucleation at the concave corner, the crystalline layer develops laterally on the substrate, with its crystallographic orientation continuously rotated. In this way, a regular spatial pattern with well-defined long-range order is eventually achieved. Our model provides a criterion to predict when such an effect becomes observable in the nucleation-mediated lateral growth. The theoretical expectations are consistent with the experimental observations.
\end{abstract}

Thin film growth has been extensively investigated in recent decades. Previous studies concentrated mostly on vertical growth (i.e., on the increase of film thickness above a substrate). ${ }^{1,2}$ To initiate a thin film growth, nevertheless, horizontal extension of individual crystalline islands on the substrate is an important step. It is known that the crystallographic orientation of an epitaxial film is well aligned with that of the substrate. Yet in some cases the crystallographic orientation of the film may become complicated. ${ }^{3-5}$ One interesting scenario is that nucleation selectively occurs at the concave corner of an expanding crystalline island and the substrate, and the island expansion (hence the thin film growth) is a repeated nucleation process at the concave corner with the orientation of each new nucleus heavily influenced by the surface tensions at the corner site. Once an embryo of nucleus (nascent nucleus) forms at the concave corner, the asymmetric local surface tensions will apply a torque to the embryo. ${ }^{6}$ Hence, the crystallographic orientation is rotated with respect to the previous nucleus. Indeed, it has been observed that, during lateral growth of $\mathrm{NH}_{4} \mathrm{Cl}$ crystallite on a glass plate, the crystallographic orientation is continuously rotated, leading to either a periodic distribution of faceted regions and roughened regions ${ }^{7}$ or a regular zigzag branches. ${ }^{8,9}$ The appearance of the specific morphology depends on the index of the initial crystalline facet contacting the substrate and the axis of rotation. ${ }^{9}$ Another example is the crystallographic wing tilt commonly observed in lateral overgrowth, ${ }^{10,11}$ which is generally ascribed to the substrate effect. The underlying

* To whom correspondence should be addressed. E-mail: muwang@ nju.edu.cn.

Nanjing University.

$\div$ Chinese Academy of Sciences.

$\S$ University of Utah.

"Oak Ridge National Laboratory.

10.1021/jp0649908 CCC: $\$ 37.00$ mechanisms of these lateral-growth-associated phenomena, however, are still not very clear, and the relationship between the rotation of the crystallographic orientation and the longrange order in aggregating crystallites has yet to be understood. In this paper, we try to establish fundamental relationships between nucleation and the rotation of crystallographic orientation. Based on previous experimental observations, we propose a model to describe the continuous rotation of crystallographic orientation. A criterion predicting the occurrence of consecutive rotation of crystallographic orientation in lateral growth is presented.

The crystallites of $\mathrm{NH}_{4} \mathrm{Cl}$ grown on a glass substrate is shown in Figure 1a. The faceted region is characterized by well-defined terraces, and the rough region is characterized by a curved and rounded top surface. Figure 1a shows that the faceted and the roughened regions appear periodically on the aggregate branch. Despite the single-crystalline appearance of the faceted crystallites, however, atomic force microscopy (AFM) shows that the terraces are in fact not parallel stacked (Figure 1b). Each terrace inclines with respect to the neighboring ones, implying that the faceted blocks are not really single-crystalline. This feature is confirmed by micro-X-ray diffraction, where elongated diffraction spots can be identified (Figure 1c). In order to pinpoint the growth mechanism, we focus on the morphology of the very front tip of the crystallite branch. Figure 1d shows that nucleation always starts from the concave corner of crystal facet and foreign substrate (glass plate), rather than on the top of the terrace. In other words, successive nucleation at the concave corner, where the nucleus contacts both the crystal facet and the foreign substrate simultaneously, acts as the step source in crystallization. For the faceted growth, it is known that the step movement controls the interfacial growth. Once the steps are continuously generated, the interfacial growth will resume. 


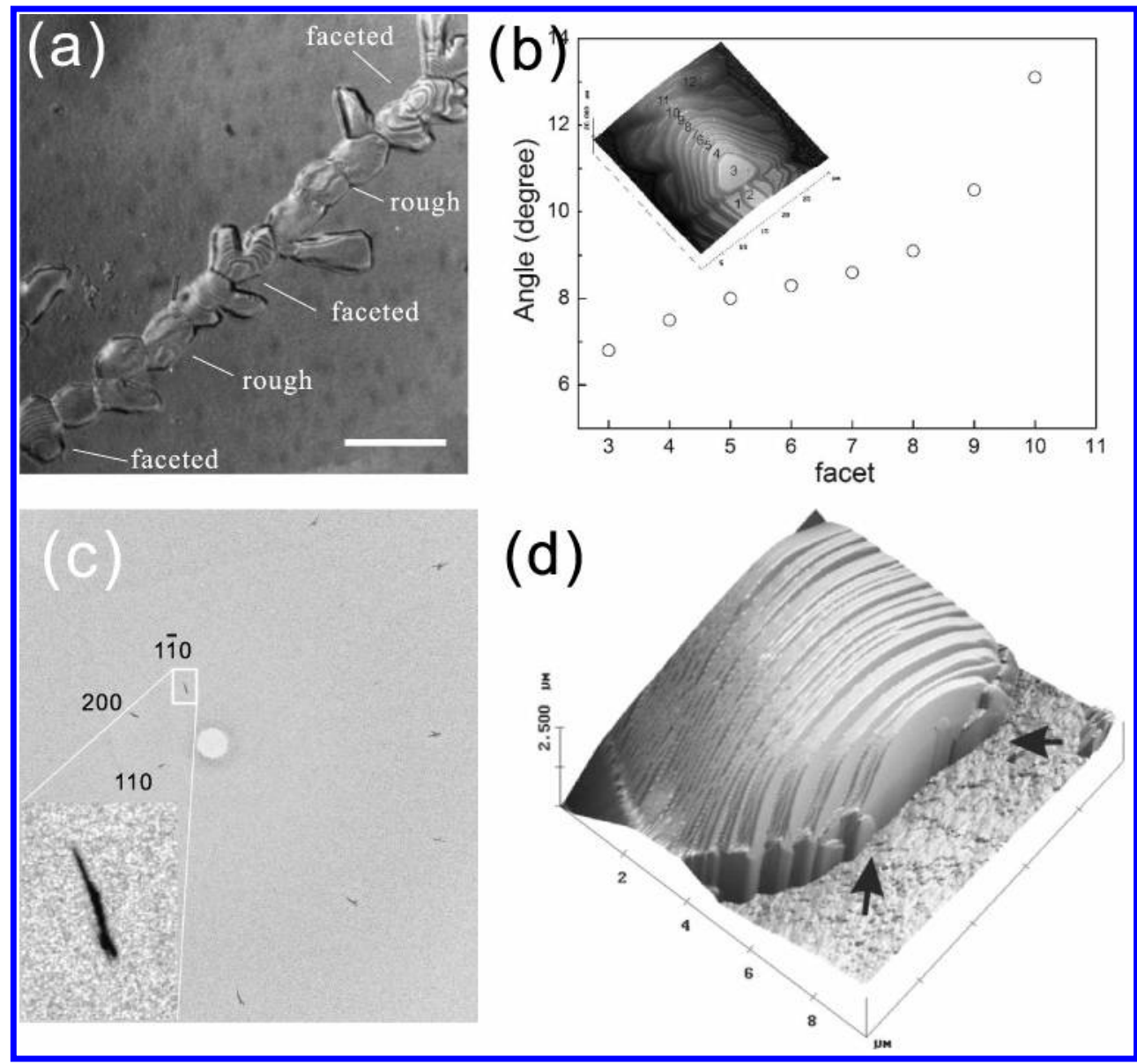

Figure 1. (a) Differential interference contrast micrograph of a crystallite aggregate of $\mathrm{NH}_{4} \mathrm{Cl}$ grown on glass plate. The faceted region and the rough region appear periodically on the branch. The bar at the corner represents $40 \mu \mathrm{m}$. Analysis indicates that the faceted face of the crystallite is (001) and the crystallite rotates its (001) face with $\langle 001\rangle$ as the axis. (b) The angle between the normal of substrate and the normal of each terrace on crystallite measured by atomic force microscope (AFM). One may find that the orientation of each step rotates serially. The inset shows the AFM topography of the faceted crystallite. (c) A micro-X-ray-diffraction pattern of a faceted crystallite. The elongation of diffraction spots can be clearly seen in the inset, which shows an enlarged diffraction spot. (d) AFM picture of the very front tip of an aggregate branch. One may find that nucleation at the concave corner of crystal fact and the substrate acts as the step source to support the layer-by-layer growth, as indicated by the arrows.

We demonstrate in our previous paper that there exists a critical driving force for crystallization, below which nucleation at the concave corner is preferred, whereas above the critical value, nucleation on flat terrace will be favored. ${ }^{7}$ The faceted feature and the favored nucleation sites shown in Figure 1 all suggest that the growth process shown here should not be a far-fromequilibrium one, although a sufficiently high supersaturation is required indeed. Since crystallographic orientation continuously rotates along a specific axis, a series of preferred growth directions appears alternatingly. In this way, a pattern with welldefined long-range-order emerges and can be observed. ${ }^{8,9}$ As we pointed out earlier, this phenomenon is not limited to the growth of $\mathrm{NH}_{4} \mathrm{Cl}$ only. A similar phenomenon has been observed in the crystallization of $\mathrm{CsCl},{ }^{12} \mathrm{Ba}\left(\mathrm{NO}_{3}\right)_{2}, \mathrm{FeSO}_{4}$, etc. ${ }^{9}$

To establish a model to describe the observed phenomena, we consider nucleation process at the concave corner of a crystal island and the foreign substrate, as schematically shown in Figure 2. Let $\gamma_{\mathrm{f}}, \gamma_{\mathrm{s}}$, and $\gamma_{\mathrm{i}}$ represent respectively the energies of three interfaces: crystal-fluid, substrate-fluid, and substratecrystal. Let $\theta_{n}$ represent the angle of the crystal facet and the substrate (here we consider lateral layer-by-layer growth, and $\theta_{n}$ is the contact angle of the $n$th layer). According to our experimental observations, successive nucleation at the concave corner acts as the step source (Figure 1d and also ref 7). The height and thickness of the nascent nucleus (embryo) are denoted

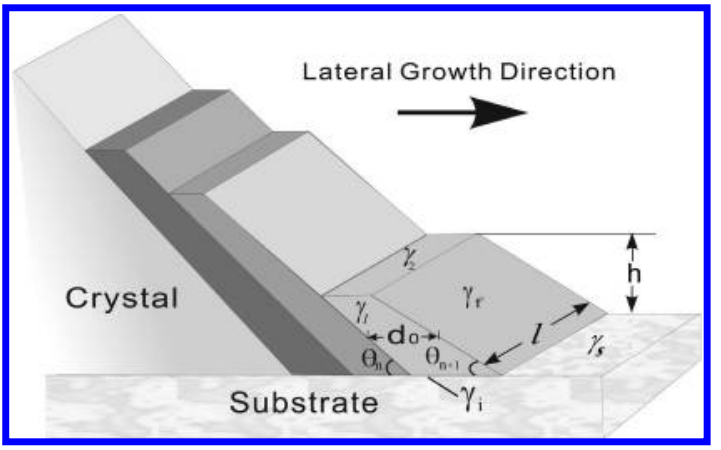

Figure 2. Schematic diagram to show successive nucleation process at the concave corner of the crystal facet (growth front) and substrate. Once an embryo is formed at the corner, the asymmetric surface/ interface tensions distort the embryo, rotate the orientation of the embryo (the front-most block with thickness $d_{0}$ ) with respect to the adjacent crystalline layers.

as $h$ and $d_{0}$, respectively. From Figure 2, one may easily find that surface energy (or surface tension) at the concave corner is asymmetric: one side of the corner is a crystalline facet, whereas the other side is the glass substrate. Therefore, once an embryo appears at the corner, the embryo will be slightly twisted with respect to the precedent layers. Consequently, a nonzero $\Delta \theta=\theta_{n+1}-\theta_{n}$ is established. According to the 
classical theory of nucleation, ${ }^{13}$ the total free energy cost to form a nucleus from a supersaturated solution basically contains three terms. The first one, $\Delta G_{\mathrm{c}}$, is a "bulk" term, which is contributed by the drop of free energy of molecules by changing from the supersaturated solution to the crystalline state. This term is negative. The second term is contributed by the surface and interface. Once the new crystalline phase is formed, this interfacial energy term $\Delta G_{\mathrm{i}}$ is added, which is always positive. Considering the rotation of crystallographic orientation, the third term, which accounts for the elastic energy originated from the mismatch (nonzero $\Delta \theta$ ) between the crystallographic orientation of the newly formed nucleus and that of the precedent layer $\left(\Delta G_{\mathrm{e}}\right)$ should be added. This term is also positive. Therefore, the total change of free energy for the formation of a nucleus can be expressed as

$$
\begin{gathered}
\Delta G=\Delta G_{\mathrm{c}}+\Delta G_{\mathrm{i}}+\Delta G_{\mathrm{e}} \\
\Delta G_{\mathrm{c}}=-\frac{h l d_{0}}{v_{\mathrm{c}}} \Delta \mu \\
\Delta G_{\mathrm{i}}=2 h d_{0} \gamma_{1}+l d_{0}\left(\gamma_{2}+\gamma_{\mathrm{i}}-\gamma_{s}\right)+l h\left(\csc \theta_{n+1}-\right. \\
\left.\csc \theta_{n}\right) \gamma_{f}+\frac{1}{2} l h\left(\cot \theta_{n+1}-\cot \theta_{n}\right)\left(\gamma_{i}-\gamma_{s}-\gamma_{2}\right) \\
\Delta G_{\mathrm{e}}=\frac{1}{2} k_{\mu}\left(\csc \theta_{n} \Delta \theta\right)^{2}\left(h l d_{0}\right)
\end{gathered}
$$

where $\Delta \mu$ is the difference of chemical potential between the crystal phase and fluid phase, $v_{\mathrm{c}}$ is the atomic volume, and $k_{\mu}$ denotes the elastic constant of the crystal. $\gamma_{1}$ and $\gamma_{2}$ represent respectively the surface energies of the side and top faces of the nucleus, as shown in Figure 2. In order that such a strained nucleation process can occur spontaneously, we expect that the elastic energy $\left(\Delta G_{\mathrm{e}}\right)$ induced by nonzero $\Delta \theta$ be compensated by decreasing the interfacial energy term $\left(\Delta G_{\mathrm{i}}\right)$ when the crystallographic orientation of the nucleus rotates with respect to the precedent layer.

For the sake of conciseness, eq 1 can be rewritten as

$$
\Delta G=-\frac{h l d_{0}}{v_{\mathrm{c}}} \Delta \mu_{\mathrm{e}}+h d_{0} \gamma_{1}+l d_{0} \gamma_{\mathrm{h}}+l h \gamma_{\mathrm{d}}
$$

with

$$
\begin{gathered}
\Delta \mu_{\mathrm{e}}=\Delta \mu-\frac{1}{2} k_{\mu} v_{\mathrm{c}}\left(h \csc \theta_{n} / d_{0}\right)^{2} \Delta \theta^{2} \\
\gamma_{\mathrm{h}}=\gamma_{2}+\gamma_{\mathrm{i}}-\gamma_{\mathrm{s}} \\
\gamma_{1}=2 \gamma_{1} \\
\gamma_{\mathrm{d}}=\left(\Delta \csc \theta_{n}\right) \gamma_{\mathrm{f}}+\frac{1}{2}\left(\Delta \cot \theta_{n}\right)\left(\gamma_{\mathrm{i}}-\gamma_{\mathrm{s}}-\gamma_{2}\right) \\
=-\left(\gamma_{f} \cos \theta_{n}+\gamma_{i}-\gamma_{s}-\gamma_{h} / 2\right) \csc ^{2} \theta_{n} \Delta \theta
\end{gathered}
$$

By minimizing the total energy for a fixed volume, the optimal aspect ratio $h / l$ is determined as $h / l=\gamma_{\mathrm{h}} / \gamma_{1}$, and the total free energy of nucleation becomes

$$
\Delta G_{0}=-\frac{h^{2} d_{0}}{v_{\mathrm{c}}} \frac{\gamma_{1}}{\gamma_{\mathrm{h}}} \Delta \mu_{\mathrm{e}}+2 h d_{0} \gamma_{1}+h^{2} \frac{\gamma_{1}}{\gamma_{\mathrm{h}}} \gamma_{\mathrm{d}}
$$

Now, in order to reach the lowest nucleation energy barrier, we obtain the rotation of crystallographic orientation by minimizing $\Delta G_{0}$ with respect to $\Delta \theta$

$$
\begin{array}{r}
\Delta \theta=\frac{d_{0}}{k_{\mu} h^{2}} \times\left[\left(\gamma_{\mathrm{f}} \cos \theta n+\gamma_{\mathrm{i}}-\gamma_{\mathrm{s}}-\gamma_{\mathrm{h}} / 2\right)-\right. \\
\left.\frac{\Delta \mu}{\gamma_{\mathrm{h}} v_{\mathrm{c}}} \frac{\mathrm{d} \gamma_{\mathrm{i}}}{\mathrm{d} \theta} d_{0} \sin ^{2} \theta_{n}\right]
\end{array}
$$

Physically, it can be understood that the rotation of the crystallographic orientation induces strain and hence increases the elastic energy. Yet this disadvantage can be compensated, as we stated earlier, by reducing the interfacial area between the crystallite and the substrate.

From eq 9 , one can find that $\Delta \theta$ is inversely proportional to the square of the embryo size $h$. This means that the embryo deformation is larger for an embryo with smaller size. On the other hand, according to nucleation theory, any embryo with the size smaller than a critical value disappears due to fluctuation, until a critical size $h^{*}$ is eventually reached. ${ }^{14}$ We suppose that when the embryo reaches size $h^{*}$ (meanwhile the embryo survives and becomes the nucleus) its crystallographic orientation is sustained, and this crystalline layer will have

$$
\begin{array}{r}
\Delta \theta^{*}=\frac{d_{0}}{k_{\mu} h^{2}}\left[\left(\gamma_{\mathrm{f}} \cos \theta_{n}+\gamma_{\mathrm{i}}-\gamma_{\mathrm{s}}-\gamma_{\mathrm{h}} / 2\right)-\right. \\
\left.\frac{\Delta \mu}{\gamma_{\mathrm{h}} v_{\mathrm{c}}} \frac{\mathrm{d} \gamma_{\mathrm{i}}}{\mathrm{d} \theta} d_{0} \sin ^{2} \theta_{n}\right]
\end{array}
$$

where $h^{*}$ is determined by maximizing $\Delta G_{0}$ with $h$, which leads to

$$
h^{*}=\frac{\gamma_{\mathrm{h}}}{\Delta \mu}
$$

Now we need to identify the sign of $\Delta \theta^{*}$. Once a nucleus is initially formed on a foreign substrate, $\gamma_{\mathrm{f}} \cos \theta_{n}^{0}+\gamma_{\mathrm{i}}-\gamma_{\mathrm{s}}=$ 0 should be satisfied. From the definition of $\gamma_{h}$ in eq 5, we know that $\gamma_{\mathrm{h}} \geq 0$. Bearing in mind that supersaturation $\Delta \mu$ is small in our experiment, eq 10 gives that $\Delta \theta^{*}$ is usually negative and increases gradually as successive nucleation sustains, until it eventually approaches zero. This means, as lateral growth proceeds layer-by-layer (Figure 2), angle $\theta_{n}$ continually decreases until an equilibrium value, $\theta_{\text {eq }}$, is reached. Thereafter the angle $\theta_{\text {eq }}$ is held. $\theta_{\text {eq }}$ is determined by $\Delta \theta^{*}=0$, according to eq 10

$$
\gamma_{\mathrm{f}} \cos \theta_{\mathrm{eq}}+\gamma_{\mathrm{i}}-\gamma_{\mathrm{s}}=\frac{\gamma_{\mathrm{h}}}{2}+\frac{\Delta \mu}{v_{\mathrm{c}}} \frac{\mathrm{d} \ln \gamma_{\mathrm{h}}}{\mathrm{d} \theta} d_{0} \sin ^{2} \theta_{\mathrm{eq}}
$$

Once $\theta_{\text {eq }}$ is reached, the crystallographic orientation of subsequently grown crystalline layers will not be changed anymore; hence, the long-range-ordering phenomena as that reported before $^{7-9}$ will not be observable on large scale. However, in some cases, before $\theta_{\mathrm{eq}}$ is reached, reconstruction of crystalline facet may take place for the sake of minimizing the total interfacial energy, and hence new contact angle appears. This is the scenario that we are going to discuss below.

It is well-known that different crystalline facets have different surface energies due to crystalline anisotropy. Yet the total free energy of a system should approach a minimum. Therefore, in crystallization once the area ratio of a facet becomes sufficiently large, it will be replaced by a few different facets with lower overall surface energy. ${ }^{15}$ This process is usually known as facet reconstruction. Following our previous discussion, as $\theta_{n}$ de- 


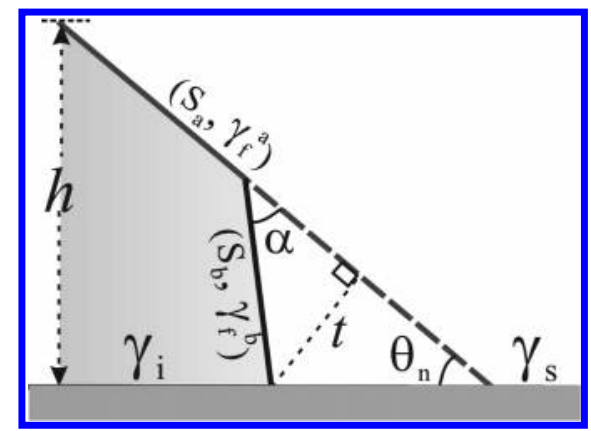

Figure 3. Schematic diagram of the side view of growth front to illustrate the decomposition of the front crystal facet from $S_{\mathrm{a}}$ to $S_{\text {a }}$ plus $S_{\mathrm{b}}$ when area $S_{\mathrm{a}}$ becomes sufficiently large.

creases, the front-most facet becomes unstable and will be decomposed to several facets. We suggest that there exists a critical angle, $\theta_{\text {cri }}$, below which reconstruction of the original facet takes place. Meanwhile, the front-most facet $S_{\mathrm{a}}$ reconstructs to $S_{\mathrm{a}}$ and $S_{\mathrm{b}}$, as illustrated in Figure 3. Let $\gamma_{\mathrm{f}}^{\mathrm{a}}$ and $\gamma_{\mathrm{f}}^{\mathrm{b}}$ denote the surface energies of $S_{\mathrm{a}}$ and $S_{\mathrm{b}}$, respectively, and the angle between $S_{\mathrm{a}}$ and $S_{\mathrm{b}}$ is $\alpha(<\pi / 2)$. In order to determine the critical angle $\theta_{\text {cri }}$, we can take account of the energy contribution of all the interfaces after reconstruction and write down the total interface energies. It follows that the total interface energy $E$ per unit length $(E / l)$ can be expressed as

$$
\begin{array}{r}
E / l=\gamma_{\mathrm{f}}^{\mathrm{a}}\left(h \csc \theta_{n}-\frac{\sin \left(\theta_{n}+\alpha\right)}{\sin \theta_{n} \sin \alpha}\right)+ \\
\gamma_{\mathrm{f}}^{\mathrm{b}} t \csc \alpha+\left(\gamma_{\mathrm{i}}-\gamma_{\mathrm{s}}\right)\left(h \cot \theta_{n}-t \csc \theta_{n}\right)
\end{array}
$$

Minimizing $E / l$ with a fixed cross section area requires that

$$
\frac{t}{h}=\frac{\left(\gamma_{\mathrm{f}}^{\mathrm{a}} \cos \theta_{n}+\gamma_{\mathrm{i}}-\gamma_{\mathrm{s}}\right) \sin \alpha-\sin \theta_{n}\left(\gamma_{\mathrm{f}}^{\mathrm{b}}-\gamma_{\mathrm{f}}^{\mathrm{a}} \cos \alpha\right)}{\left(\gamma_{\mathrm{i}}-\gamma_{\mathrm{s}}+\gamma_{\mathrm{f}}^{\mathrm{a}} / \cos \theta_{n}\right) \sin \left(\theta_{n}+\alpha\right)}
$$

According to eq 14, when $\theta_{n}$ is sufficiently large, $t / h<0$ (unphysical), meaning that the facet $S_{\mathrm{b}}$ has not yet emerged. As the growth front moves on, $S_{\mathrm{a}}$ inclines continuously. Thus the area of $S_{\mathrm{a}}$, and hence the total surface energy, both increase accordingly. Once $\theta_{n}$ becomes smaller than $\theta_{\text {cri }}, t / h>0$, indicating that reconstruction of facet $S_{\mathrm{a}}$ occurs and facet $S_{\mathrm{b}}$ emerges. Therefore, the critical angle $\theta_{\text {cri }}$ can be determined by $t / h=0$. It follows that

$$
\frac{\cos \theta_{\text {cri }}+\left(\gamma_{\mathrm{i}}-\gamma_{\mathrm{s}}\right) / \gamma_{\mathrm{f}}^{\mathrm{a}}}{\sin \theta_{\mathrm{cri}}}=\frac{\gamma_{\mathrm{f}}^{\mathrm{b}} / \gamma_{\mathrm{f}}^{\mathrm{a}}-\cos \alpha}{\sin \alpha}
$$

When $S_{\mathrm{b}}$ emerges, it develops and becomes the dominating facet on the growing front. $S_{\mathrm{b}}$ has a new and larger contact angle. For the reasons discussed in the above paragraphs, this angle decreases for subsequent layers as the layer-by-layer growth continues. Similar to what happened to $S_{\mathrm{a}}$, for $S_{\mathrm{b}}$ there exists a new equilibrium contact angle, $\theta_{\text {eq }}^{\prime}$, and a new critical angle $\theta_{\text {cri }}^{\prime}$, which together define the criterion for the emergence of new facet $S_{\mathrm{c}}$. Once $\theta_{\text {eq }}^{\prime}<\theta_{\text {cri }}^{\prime}$ is satisfied, the facet reconstruction takes place again. This process may continue infinitely. Practically, however, due to the limitation of the available lowenergy facets and the symmetry of crystalline structure, when a crystallite rotates along a selected axis, the available facets are usually very limited. For example, for an fcc crystal, when it rotates with $\langle 100\rangle$ as the axis, the possible low-energy facets along the growth direction could be (001) and (110). As a result, the crystallographic orientation of the growth front alternates between $S_{\mathrm{a}}$ (possibly (001)) and $S_{\mathrm{b}}$ (possibly (110)). Therefore, via the self-supporting facet reconstruction, continuous rotation of crystallographic orientation sustains.

Combining eqs 12 and 15 and condition $\theta_{\text {eq }}<\theta_{\text {cri, }}$, one obtains

$$
\frac{\gamma_{\mathrm{f}}^{\mathrm{b}}-\gamma_{\mathrm{f}}^{\mathrm{a}} \cos \alpha}{\sin \alpha}<\frac{\gamma_{\mathrm{h}}}{2 \sin \theta_{\mathrm{eq}}}+\frac{\Delta \mu}{\gamma_{\mathrm{h}} v_{\mathrm{c}}} \frac{\mathrm{d} \gamma_{\mathrm{i}}}{\mathrm{d} \theta} d_{0} \sin \theta_{\mathrm{eq}}
$$

Equation 16 is the criterion to achieve continuous rotation of crystallographic orientation in lateral growth. Equation 16 can be more easily satisfied for larger $\gamma_{\mathrm{h}}$, larger positive $\mathrm{d}\left(\ln \gamma_{\mathrm{h}}\right) /$ $\mathrm{d} \theta$, and/or higher supersaturation.

The above theoretical expectations are supported by experiments. First, as indicated by Figure 1c, each X-ray diffraction spot is elongated along specific directions. The limited elongation of the diffraction spots indicates that the crystallographic orientation has been rotated continuously for limited degrees with a well defined axis. Second, accumulation of strain in crystallite, which is induced by successive rotation of crystallographic orientation, will trigger a morphological instability known as Grinfeld instability. ${ }^{16-19}$ Due to this instability, a strained crystallite will be separated when a critical size is reached in order to release the elastic energy. ${ }^{20}$ The average size of the separated crystallites can be estimated from the characteristic length of Grinfeld instability $\lambda$

$$
\lambda=\frac{\pi \gamma_{2}\left(1-\sigma^{2}\right)}{k_{\mu} \epsilon^{2}}=\frac{\pi \gamma_{2}\left(1-\sigma^{2}\right)}{k_{\mu}}\left(\frac{d_{0}}{h^{*} \csc \theta_{n} \Delta \theta^{*}}\right)^{2}
$$

where $h^{*}$ is the height of the critical nucleus, $\Delta \theta^{*}$ is the corresponding rotation angle of the critical nucleus, and $\epsilon$ is the internal strain. Based on the experimental observations, we estimate $\Delta \theta$ to be of the order of $10^{-5}$ degree, and the strain to be of the order of $1.8 \times 10^{-3}$. $^{7}$ This value, together with other material parameters of $\mathrm{NH}_{4} \mathrm{Cl},{ }^{21}$ yields $\lambda=36 \mu \mathrm{m}$. This means that when the size of a crystallite reaches $\lambda$, its top surface will become unstable and wrinkles may develop to separate the crystallite. The crystallite size will be of the order of $\lambda / 2$. In our experiments, the crystallite size is about $20 \mu \mathrm{m}$, which is in excellent agreement with theoretical expectation. Further, within a spatial period of faceted-rough regions, the overall rotated angle corresponding to the spatial periodicity, $\Delta \Theta$, is determined by the crystal structure and the axis of rotation. It can be estimated that the number of separated crystallites within each spatial period should be $n \simeq d_{0}(\Delta \Theta / \Delta \theta) / \lambda$. Combining eqs 10,11 , and $17, n$ can be expressed as

$$
\begin{aligned}
n & \simeq \frac{\Delta \Theta k_{\mu} h^{2} \Delta \theta}{\pi \gamma_{2}\left(1-\sigma^{2}\right) d_{0} \sin ^{2} \theta_{n}} \\
& \simeq \frac{\Delta \Theta\left(\gamma_{\mathrm{f}} \cos \theta_{n}+\gamma_{\mathrm{i}}-\gamma_{\mathrm{s}}-\gamma_{\mathrm{h}} / 2\right)}{\pi \gamma_{2}\left(1-\sigma^{2}\right) \sin ^{2} \theta_{n}}
\end{aligned}
$$

Clearly the number of separated crystallites within each spatial period, $n$, is determined by the intrinsic properties of the growth system, and is independent of supersaturation $\Delta \mu$. This is indeed in agreement qualitatively with our experimental observations. As shown in Figure 1a, as well as in ref 7, there exists a periodic distribution of faceted region and rough region on the crystallite branches. Experimentally, we find that when the driving force of crystallization is low the average crystallite size becomes larger, and meanwhile, the spatial period of the faceting- 


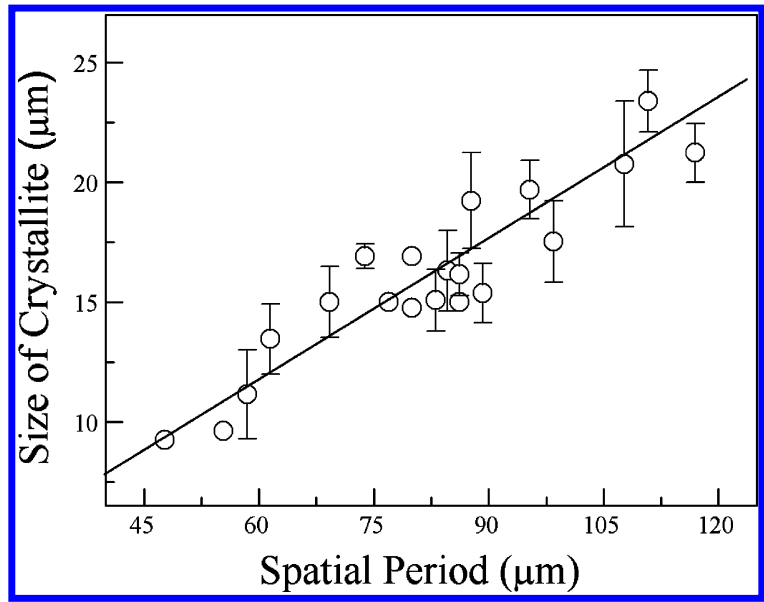

Figure 4. Plot to show the average crystallite size as a function of spatial period in the aggregate (the different spatial periodicity is achieved by carrying out experiments with different supersaturation). The linear fit indicates that the number of crystallites within each spatial period is independent of the driving force of crystallization.

roughening transition becomes longer. When the driving force of crystallization is high, the nucleation rate is high. Hence, the crystal branches grow faster, yet the size of each crystallite becomes smaller, and meanwhile the spatial period on the branch becomes shorter. We made statistics on the experimental observations and plotted the result in Figure 4, which demonstrates the experimentally observed spatial period of the facetingroughening distribution on the aggregate branch as a function of average crystallite size. The data can be linearly fit, and the slope corresponds to the number of crystallites within each period. The linear fitting in Figure 4 means that the number of crystallites within each spatial period does not depend on the driving force of crystallization, although the crystallite size does, which is exactly predicted by eq 18 . Figure 4 strongly suggests that the phenomena shown in Figure 1 are caused by continuous rotation of crystallographic orientation in crystallization and the associated strain accumulated in the aggregates.

In conclusion, we establish a theoretical model to interpret a new lateral growth behavior driven by successive nucleation at the concave corner of the crystal facet and the foreign substrate. Due to the asymmetry of surface tensions at the concave corner, the nascent nucleus is strained and the crystallographic orienta- tion is successively rotated. The criterion for the occurrence of the continuous rotation of crystallographic orientation is derived, and the theoretical predictions agree with experimental observations. Since asymmetric surface energies at the concave corner of the crystal facet and the foreign substrate always exist in heteroepitaxial growth, and nucleation at the reentrant corner is thermodynamically favored, we suggest that the growth model presented here is an important complement to our current understanding of thin film growth.

Acknowledgment. The authors acknowledge the financial support from the Ministry of Science and Technology of China (2004CB619005) and the National Science Foundation of China (10021001, 10374042, and 10374043). Authors also thank V. Fleury, P. Bennema, and M. Plapp for enlightening discussions.

\section{References and Notes}

(1) Handbook of Crystal Growth: Thin Films and Epitaxy; Hurle, D. T. J., Ed.; North-Holland: Amsterdam, 1994.

(2) Nucleation \& Growth of Thin Films; Lewis, B., Anderson, J. C., Eds.; Academic Press: New York, 1997.

(3) Strom, C. S.; Liu, X. Y.; Wang, M. J. Phvs. Chem. B 2000, 104, $9638-9646$.

(4) Liu, X. Y.; Strom, C. S. J. Chem. Phvs. 2000, 113, 4408

(5) Liu, X. Y.; Wang, M.; Liu, D. W. et al. J. Cryst. Growth 2000, $208,687$.

(6) The example to show the effect of the torque induced by asymmetric interfacial tensions on nanocrystalline domain has been given by Moldovan, D.; Yamakov, V.; Wolf, D.; Philipot, S. R. Phvs. Rev. Lett. 2002, 89, 106101.

(7) Wang, Mu. et al. Phvs. Rev. Lett. 2005, 94, 125505.

(8) Wang, Mu. et al. Phvs. Rev. Lett. 1998, 80, 3089

(9) Li, D. W. et al. J. Phvs. Chem. B 2003, 107, 96.

(10) Fini, P. et al. Appl. Phys. Lett. 1999, 75, 1706; 2000, 76, 3893

(11) Dürr, A. C. et al. Phvs. Rev. Lett. 2003, 90, 16104.

(12) Pan, W.; Mao, Y. W.; Shu, D. J. et al., to be published.

(13) Kelton, K. F. Solid State Physics; Academic: New York, 1991; Vol. 45, pp 75-90.

(14) The volume of an embryo depends on $d_{0}, h$, and $l$. Whereas $h$ and $l$ are correlated as $h / l=\gamma_{\mathrm{h}} / \gamma_{1}$ and $d_{0}$ is a constant, therefore the volume of an embryo can be determined by $h$.

(15) Handbook of Surface Science, Vol. 1, Physical Structure; Unertl,

W. N., Ed.; Elsevier Science: Amsterdam, 1996; pp 51-99.

(16) Asaro, R. J.; Tiller, W. A. Metall. Trans. 1972, 3, 1789.

(17) Grinfeld, M. A. Sov. Phys. Dokl. 1986, 31, 831.

(18) Berrhar, J. et. al. Phvs. Rev. B 1992, 46, 13487.

(19) Yang, W. H.; Srolovitz, D. J. Phys. Rev. Lett. 1993, 71, 1593.

(20) Muller, J. Ph.D. thesis, McGill University: Montreal, Quebec, Canada, 1998

(21) Winkler, B. et al. J.Phvs.: Condens. Matter 2000, 12, 2093. 